\title{
Article \\ Synergistic Combination of AS101 and Azidothymidine against Clinical Isolates of Carbapenem-Resistant Klebsiella pneumoniae
}

\author{
Chung-Lin Sung ${ }^{1}$, Wei-Chun Hung ${ }^{2}$, Po-Liang Lu ${ }^{3,4,5} \mathbb{D}$, Lin Lin ${ }^{6}$, Liang-Chun Wang ${ }^{7} \mathbb{D}$, Tsung-Ying Yang ${ }^{1,3}, * \mathbb{D}$ \\ and Sung-Pin Tseng 1,7,8,9,*
}

1 Department of Medical Laboratory Science and Biotechnology, College of Health Sciences, Kaohsiung Medical University, Kaohsiung 807, Taiwan; jimmy0922236561@gmail.com

2 Department of Microbiology and Immunology, College of Medicine, Kaohsiung Medical University, Kaohsiung 807, Taiwan; wchung@kmu.edu.tw

3 Center for Liquid Biopsy and Cohort Research, Kaohsiung Medical University, Kaohsiung 807, Taiwan; d830166@gmail.com

4 Division of Infectious Diseases, Department of Internal Medicine, Kaohsiung Medical University Hospital, Kaohsiung 807, Taiwan

5 School of Post-Baccalaureate Medicine, College of Medicine, Kaohsiung Medical University, Kaohsiung 807, Taiwan

6 Department of Culinary Art, I-Shou University, Kaohsiung 84001, Taiwan; lynnlin@isu.edu.tw

7 Department of Marine Biotechnology and Resources, National Sun Yat-sen University, Kaohsiung 804, Taiwan; marknjoy@g-mail.nsysu.edu.tw

check for updates

Citation: Sung, C.-L.; Hung, W.-C.; Lu, P.-L.; Lin, L.; Wang, L.-C.; Yang,

T.-Y.; Tseng, S.-P. Synergistic

Combination of AS101 and

Azidothymidine against Clinical Isolates of Carbapenem-Resistant Klebsiella pneumoniae. Pathogens 2021, 10, 1552. https://doi.org/10.3390/ pathogens10121552

Academic Editor: Lawrence S. Young

Received: 23 October 2021

Accepted: 25 November 2021

Published: 29 November 2021

Publisher's Note: MDPI stays neutral with regard to jurisdictional claims in published maps and institutional affiliations.

Copyright: (c) 2021 by the authors. Licensee MDPI, Basel, Switzerland. This article is an open access article distributed under the terms and conditions of the Creative Commons Attribution (CC BY) license (https:/ / creativecommons.org/licenses/by/ $4.0 /)$.
8 Drug Development and Value Creation Research Center, Kaohsiung Medical University, Kaohsiung 807, Taiwan

9 Graduate Institute of Animal Vaccine Technology, College of Veterinary Medicine, National Pingtung University of Science and Technology, Pingtung 912, Taiwan

* Correspondence: zegma040899@gmail.com (T.-Y.Y.); tsengsp@kmu.edu.tw (S.-P.T.); Tel.: +886-7-312-1101 (ext. 2007) (T.-Y.Y.); +886-7-312-1101 (ext. 2350) (S.-P.T.)

\begin{abstract}
Owing to the over usage of carbapenems, carbapenem resistance has become a vital threat worldwide, and, thus, the World Health Organization announced the carbapenem-resistant Enterobacteriaceae (CRE) as the critical priority for antibiotic development in 2017. In the current situation, combination therapy would be one solution against CRE. Azidothymidine (AZT), a thymidine analog, has demonstrated its synergistically antibacterial activities with other antibiotics. The unexpected antimicrobial activity of the immunomodulator ammonium trichloro(dioxoethylene- $0, o^{\prime}$ )tellurate (AS101) has been reported against carbapenem-resistant Klebsiella pneumoniae (CRKP). Here, we sought to investigate the synergistic activity between AS101 and AZT against 12 CRKP clinical isolates. According to the gene detection results, the bla $a_{\mathrm{OXA}-1}(7 / 12,58.3 \%), b l a_{\mathrm{DHA}}(7 / 12,58.3 \%)$, and $b a_{\mathrm{KPC}}(7 / 12,58.3 \%)$ genes were the most prevalent ESBL, AmpC, and carbapenemase genes, respectively. The checkerboard analysis demonstrated the remarkable synergism between AS101 and AZT, with the observable decrease in the MIC value for two agents and the fractional inhibitory concentration (FIC) index $\leq 0.5$ in all strains. Hence, the combination of AS101 and azidothymidine could be a potential treatment option against CRKP for drug development.
\end{abstract}

Keywords: carbapenem-resistant Enterobacteriaceae (CRE); synergistic activity; AS101; azidothymidine

\section{Introduction}

Carbapenem-resistant Enterobacteriaceae (CRE) was announced as the critical priority for antibiotic development in 2017 [1]. Recently, the COVID-19 pandemic promoted the spread of carbapenem-resistant Klebsiella pneumoniae (CRKP) [2], highlighting an urgent need for novel treatment options. The high carbapenem-resistant rates were revealed in some parts of Europe [3], especially Southern Europe. According to the European Centre 
for Disease Prevention and Control EARS-Net, 58.3\% of 312 Greek K. pneumoniae isolates collected in 2019 were resistant to either imipenem or meropenem [4]. In Romania, 32.3\% of $470 \mathrm{~K}$. pneumoniae were reported as carbapenem-resistant isolates; in Italy, $28.5 \%$ of 7327 K. pneumoniae were defined as carbapenem-resistant isolates. A regional resistance surveillance program in Asia-Pacific reported 25\% carbapenem-resistant rate in K. pneumoniae for India and 5\% for both the Philippines and Thailand [5]. A more recent surveillance conducted in Taiwan, 2018, described a 7.2\% carbapenem-resistant rate in 346 K. pneumoniae isolates [6]. In view of the aforementioned reports, the development of novel therapeutic options to address CRKP is urgently needed.

In recent years, some novel $\beta$-lactam- $\beta$-lactamase inhibitor combination therapies have been launched in clinical settings, for instance, meropenem-vaborbactam, imipenemrelebactam, and ceftazidime-avibactam [7-10]. Moreover, azidothymidine was reported to show synergistic activities with some clinical antimicrobial agents such as colistin and fosfomycin. [11-13]. Azidothymidine (AZT), a thymidine analog, possesses an antiretroviral activity and clinically treats patients with human immunodeficiency virus (HIV) infections, the acquired immunodeficiency syndrome (AIDS) [14]. Lately, studies have reported that azidothymidine combined with antibiotics produced synergistic activities against antibiotic-resistant gram-negative bacteria both in vitro and in vivo [11]. A previous study also reported that a combination of colistin and azidothymidine revealed synergistic activity against colistin-resistant CRKP clinical isolates [12]. In a phase I clinical trial for the combination of AZT and colistin, the results revealed that a dosage of 2 million IU CMS plus $100 \mathrm{mg}$ AZT twice a day might be sufficient for urinary tract infections (UTIs) [15]. Although the phase I clinical trial of the combination of AZT and colistin is in progress, colistin could be a temporary option for CRKP treatment but could not be permanent because of its nephrotoxicity and neurotoxicity [16].

AS101 is a fully-synthesized, tellurium-containing, organic compound with a small molecular weight of 312 Daltons [17,18]. With its characteristic of immunomodulation, AS101 was used to treat autoimmune diseases, inflammatory bowel disease (IBD), multiple sclerosis (MS), and psoriasis [19], and some of its applications are in ongoing clinical trials. Moreover, AS101 demonstrated anti-inflammatory activity [19], antiviral activities (HIV-1 and WNV) [20,21], and antimicrobial activities (carbapenem-resistant Acinetobacter baumannii, K. pneumoniae, Enterobacter cloacae) [22-25]. In a recent study, Yang et al. demonstrated its in vitro and in vivo antimicrobial activity against carbapenem-resistant Acinetobacter baumannii [22]. Although previous efforts posed that AS101 could be a potential option to treat CRKP, our study described that the MIC values of AS101 against CRKP was up to $32 \mu \mathrm{g} / \mathrm{mL}$ [23], which is not far away from its $50 \%$ cytotoxicity level $(145 \mu \mathrm{g} / \mathrm{mL})$ [21]. Hence, it might be unsafe for high dose. To this end, some improvements might be needed to increase the antibacterial activity of AS101. Accordingly, finding an alternative agent to combine with AZT for CRKP treatment is of vital importance. Given the previous efforts on AZT combinations and AS101, we sought to evaluate the synergistic activities of AZT plus AS101 against CRKP in this study.

\section{Results}

\subsection{Antimicrobial Susceptibility Testing}

Among the 12 isolates tested in this study, over $80 \%$ of resistant rates were observed in 16 agents (Table 1): ampicillin (12/12, 100\%), aztreonam (10/12, 83.3\%), ceftazidime $(11 / 12,91.7 \%)$, cefazolin $(12 / 12,100 \%)$, ciprofloxacin $(12 / 12,100 \%)$, cefepime $(10 / 12$, $83.3 \%)$, cefoxitin $(12 / 12100 \%)$, ceftriaxone $(12 / 12,100 \%)$, cefotaxime $(11 / 12,91.7 \%)$, imipenem $(12 / 12,100 \%)$, levofloxacin (12/12, 100\%), meropenem (12/12, 100\%), trimethoprim/sulfamethoxazole $(11 / 12,91.7 \%)$, piperacillin-tazobactam $(11 / 12,91.7 \%)$, ertapenem $(12 / 12,100 \%)$, doripenem $(12 / 12,100 \%)$. In contrast to the 16 agents with low susceptibilities, tigecycline, amikacin, and gentamicin had higher susceptibilities of $91.7 \%(11 / 12)$, $66.7 \%(8 / 12)$, and $41.7 \%(5 / 12)$, respectively. 
Table 1. Antimicrobial susceptibilities for 19 antimicrobial agents.

\begin{tabular}{cccc}
\hline \multirow{2}{*}{ Antimicrobial Agent } & \multicolumn{3}{c}{ Antibiotic Susceptibility } \\
\cline { 2 - 4 } & $\mathbf{S}$ & $\mathbf{I}$ & $\mathbf{R}$ \\
\hline Amikacin & $66.7 \%$ & $0.0 \%$ & $33.3 \%$ \\
Ampicillin & $0.0 \%$ & $0.0 \%$ & $100.0 \%$ \\
Aztreonam & $16.7 \%$ & $0.0 \%$ & $83.3 \%$ \\
Ceftazidime & $0.0 \%$ & $8.3 \%$ & $91.7 \%$ \\
Cefazolin & $0.0 \%$ & $0.0 \%$ & $100.0 \%$ \\
Ciprofloxacin & $0.0 \%$ & $0.0 \%$ & $100.0 \%$ \\
Cefepime & $0.0 \%$ & $16.7 \%$ & $83.3 \%$ \\
Cefoxitin & $0.0 \%$ & $0.0 \%$ & $100.0 \%$ \\
Ceftriaxone & $0.0 \%$ & $0.0 \%$ & $100.0 \%$ \\
Cefotaxime & $8.3 \%$ & $0.0 \%$ & $91.7 \%$ \\
Gentamicin & $41.7 \%$ & $0.0 \%$ & $58.3 \%$ \\
Imipenem & $0.0 \%$ & $0.0 \%$ & $100.0 \%$ \\
Levofloxacin & $0.0 \%$ & $0.0 \%$ & $100.0 \%$ \\
Meropenem & $0.0 \%$ & $0.0 \%$ & $100.0 \%$ \\
Piperacillin-Tazobactam & $8.3 \%$ & $0.0 \%$ & $91.7 \%$ \\
Ertapenem & $8.3 \%$ & $0.0 \%$ & $91.7 \%$ \\
Tigecycline & $0.0 \%$ & $0.0 \%$ & $100.0 \%$ \\
Doripenem & $91.7 \%$ & $8.3 \%$ & $0.0 \%$ \\
Trimethoprim/Sulfamethoxazole & $0.0 \%$ & $0.0 \%$ & $100.0 \%$
\end{tabular}

1, abbreviations: S, susceptible; I, intermediate-resistant; $R$, resistant.

\subsection{Detection of Resistance Gene}

Among the 12 CRKP isolates, the Klebsiella pneumoniae carbapenemase (KPC) gene was the dominant carbapenemase gene detected in seven isolates $(7 / 12,58.3 \%)$, followed by $b l a_{\mathrm{OXA}}-48$ gene in one isolate $(1 / 12,8.3 \%)$ (Figure 1$)$. Four of the isolates $(4 / 12,33.3 \%)$ were found with only one type of $\beta$-lactamase genes, mostly $b l a_{\mathrm{KPC}}$ gene (three isolates). Nevertheless, only one of the isolates $(1 / 12,8.3 \%)$ had two types of resistant gene (bla OXA-48 and $\left.b l a_{\mathrm{OXA}-1}\right)$. Two of the isolates $(2 / 12,16.7 \%)$ harbored three types of resistant gene, including either $b l a_{\mathrm{OXA}-1}$ or $b l a_{\mathrm{CTX}-\mathrm{M}}$ in one of the isolates, and $b l a_{\mathrm{DHA}}$ and $b l a_{\mathrm{TEM}}$ in both. In addition, five isolates $(5 / 12,41.6 \%)$ were found with four types of $\beta$-lactamase genes simultaneously. All of these five isolates harbored $b l a_{\mathrm{OXA}-1}$ and $b l a_{\mathrm{DHA}}$. Moreover, four of these five isolates harbored $b l a_{\mathrm{SHV}-12}$ and $b l a_{\mathrm{KPC}}$. The $b l a_{\mathrm{DHA}}$ gene was detected in seven isolates, but none of the isolates harbored $b a_{\mathrm{CMY}}$. The $b \operatorname{la}_{\mathrm{OXA}-1}$ gene was the most prevalent of the ESBL genes $(7 / 12,58.3 \%)$ detected in this study, followed by bla $a_{\mathrm{TEM}}(4 / 12,33.3 \%)$, bla $_{\mathrm{SHV}-12}(4 / 12,33.3 \%)$, and bla $a_{\mathrm{CTX}-\mathrm{M}}(2 / 12,16.7 \%)$.

\subsection{Evaluation of Synergistic Effects}

The MIC values for AS101 against the 12 CRKP isolates ranged from 2 to $512 \mu \mathrm{g} / \mathrm{mL}$ (Table 2), with the $\mathrm{MIC}_{50}, \mathrm{MIC}_{75}$, and $\mathrm{MIC}_{90}$ of 128,256 , and $512 \mu \mathrm{g} / \mathrm{mL}$, respectively. The MIC range of azidothymidine (AZT) against the 12 CRKP was from 0.5 to $4 \mu \mathrm{g} / \mathrm{mL}$, and the $\mathrm{MIC}_{50}, \mathrm{MIC}_{75}$, and $\mathrm{MIC}_{90}$ were revealed as 1,2 , and $2 \mu \mathrm{g} / \mathrm{mL}$, respectively. With the combination of AS101 and AZT, noticeable decreases for $\mathrm{MIC}_{50}, \mathrm{MIC}_{75}$, and $\mathrm{MIC}_{90}$ were observed in both AS101 (from 128, 256, and 512, respectively, to 8, 16, and $16 \mu \mathrm{g} / \mathrm{mL}$ ) and AZT (from 1, 2, and 2, respectively, to $0.25,0.25$, and $0.5 \mu \mathrm{g} / \mathrm{mL}$ ) (Table 2). The MIC distributions of AS101 and AZT alone or in combination are visualized in Figure 2. Significant changes in MIC distributions were noted for AS101 and AZT (both $p<0.0001$ ), with decreased MIC ranges for AS101 (from 2-12 to 0.5-32 $\mu \mathrm{g} / \mathrm{mL}$ ) and AZT (from 0.5-4 to $0.0625-1 \mu \mathrm{g} / \mathrm{mL}$ ) (Table 2). The fractional inhibitory concentration (FIC) indexes of the 12 isolates were all $\leq 0.5$ (Table 3), suggesting the synergistic interaction between AS101 and azidothymidine. Additionally, the synergistic effect was observed in the growth curve (Figure S1.) Supported by the aforementioned results, the combination therapy of AS101 and AZT is a potential treatment option for CRKP infections, needing further investigation 
in future study. All of the checkerboard methods for checking the synergistic effect were performed and repeated three times, and all results were reproducible.

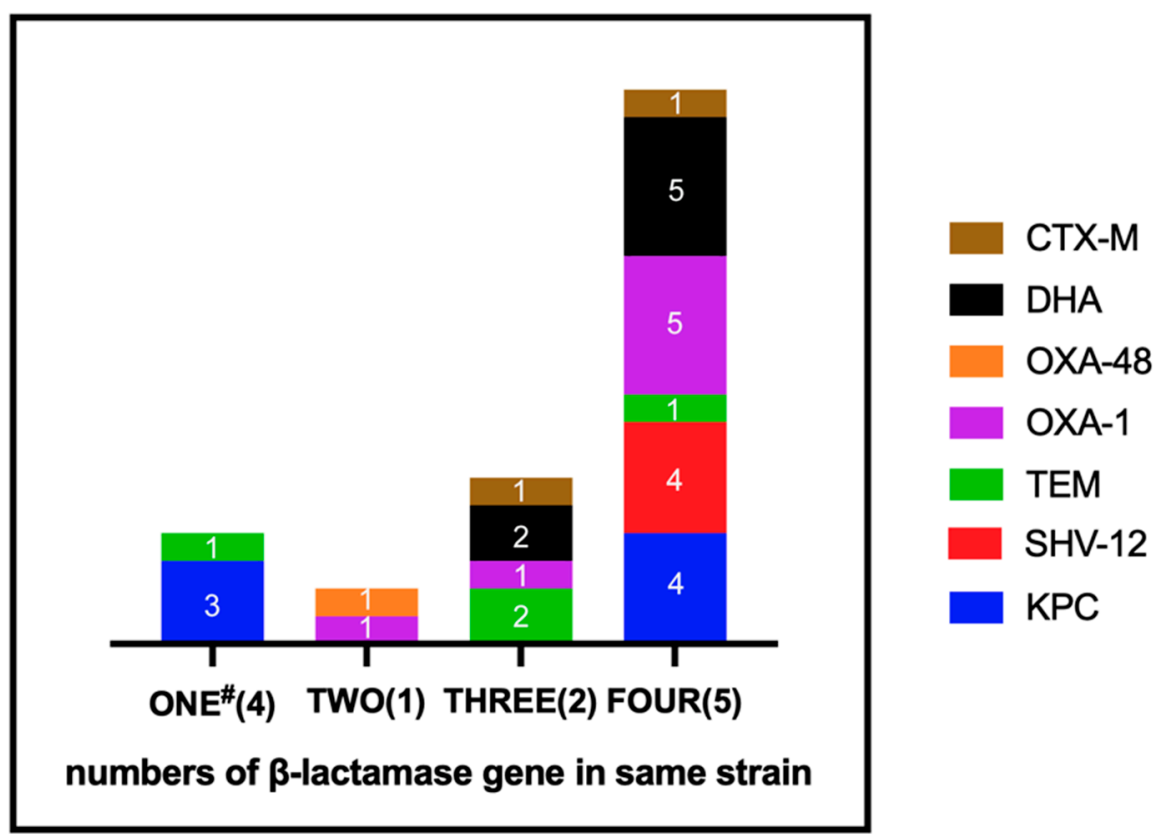

Figure 1. Distribution of the resistant mechanism among 12 isolates. \#, number of resistant mechanisms on the same strain simultaneously (number of isolates).

Table 2. MIC values range, $\mathrm{MIC}_{50}, \mathrm{MIC}_{75}, \mathrm{MIC}_{90}$ for AS101, azidothymidine, and AS101azidothymidine combination.

\begin{tabular}{ccccccccc}
\hline \multirow{2}{*}{ Agent } & \multicolumn{3}{c}{ MIC Alone $^{*}(\mu \mathrm{g} / \mathrm{mL})$} & \multicolumn{3}{c}{ MIC in Combination ${ }^{*}(\mu \mathrm{g} / \mathrm{mL})$} \\
\cline { 2 - 9 } & Range & MIC $_{\mathbf{5 0}}$ & MIC $_{\mathbf{7 5}}$ & MIC $_{\mathbf{9 0}}$ & Range & MIC $_{\mathbf{5 0}}$ & MIC $_{\mathbf{7 5}}$ & MIC $_{\mathbf{9 0}}$ \\
\hline AS101 & $2-512$ & 128 & 256 & 512 & $0.5-32$ & 8 & 16 & 16 \\
AZT & $0.5-4$ & 1 & 2 & 2 & $0.0625-1$ & 0.25 & 0.25 & 0.5 \\
\hline
\end{tabular}

Abbreviations: MIC, minimum inhibitory concentration; AZT azidothymidine; $\mathrm{MIC}_{50}$, 50th percentile of MIC MIC75, 75th percentile of MIC; MIC 90 , 90th percentile of MIC. *, MIC for AS101 or AZT in single agent; \#, MIC for combination of AS101 and AZT.
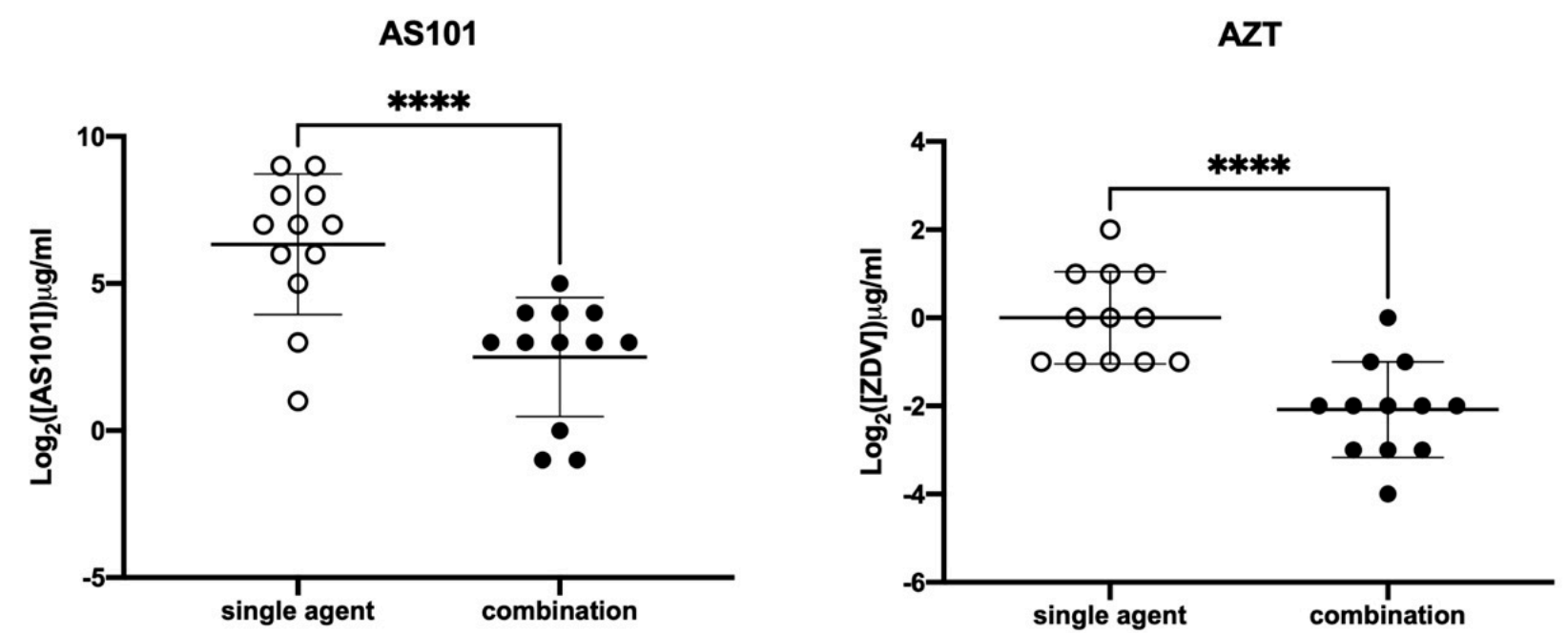

Figure 2. MIC distributions of AS101 (hollow circles), azidothymidine (AZT, hollow circles), and their combination (filled circles); bars in the middle of circles indicated means and standard deviations. ${ }^{* * *}, p<0.0001$ with the paired $t$-test. 
Table 3. MIC values and FICI of 12 isolates against AS101, azidothymidine, AS101-azidothymidine combination.

\begin{tabular}{|c|c|c|c|c|c|c|}
\hline \multirow{2}{*}{ Strains } & \multicolumn{2}{|c|}{ MIC Alone * $(\mu \mathrm{g} / \mathrm{mL})$} & \multicolumn{2}{|c|}{ MIC in Combination ${ }^{\#}(\mu \mathrm{g} / \mathrm{mL})$} & \multirow{2}{*}{$\frac{\text { FICI }}{(\mathrm{AS} 101, \mathrm{AZT})}$} & \multirow{2}{*}{ Interpretation } \\
\hline & AS101 & AZT & AS101 & AZT & & \\
\hline CRE-918 & 128 & 0.5 & 0.5 & 0.25 & 0.5 & synergistic \\
\hline CRE-949 & 256 & 1 & 8 & 0.25 & 0.28 & synergistic \\
\hline CRE-1017 & 64 & 2 & 8 & 0.5 & 0.38 & synergistic \\
\hline CRE-1038 & 256 & 0.5 & 16 & 0.125 & 0.31 & synergistic \\
\hline CRE-1044 & 512 & 0.5 & 16 & 0.0625 & 0.16 & synergistic \\
\hline CRE-1085 & 32 & 2 & 8 & 0.25 & 0.38 & synergistic \\
\hline CRE-1086 & 128 & 2 & 16 & 0.5 & 0.38 & synergistic \\
\hline CRE-1125 & 2 & 1 & 0.5 & 0.25 & 0.5 & synergistic \\
\hline CRE-1136 & 128 & 1 & 32 & 0.25 & 0.5 & synergistic \\
\hline CRE-1290 & 64 & 0.5 & 8 & 0.125 & 0.38 & synergistic \\
\hline CRE-1382 & 512 & 0.5 & 8 & 0.125 & 0.27 & synergistic \\
\hline CRE-1536 & 8 & 4 & 1 & 1 & 0.38 & synergistic \\
\hline
\end{tabular}

Abbreviations: MIC, minimum inhibitory concentration; AZT, azidothymidine; FICI, fractional inhibitory concentration index. *, MIC for AS101 or AZT in single agent; ", MIC for combination of AS101 and AZT.

\section{Discussion}

Due to the transmission of resistant genes, the prevalence of carbapenem-resistant Enterobacteriaceae (CRE) has become a deadly threat to public health in the past years, causing severe infections associated with significant mortality [26]. Among approximately 140,000 cases of healthcare-associated Enterobacteriaceae infections in the United States yearly, around 9300 instances were caused by CRE, and 520 patients infected by CRE died [27]. The multidrug resistance was usually noticed among CRE isolates [28-31]. In a previous study conducted in the USA, a larger amount of CRKP isolates were collected from Jan 2014 to Mar 2015 in a long-term acute care hospital network [30]. The susceptibilities of selected antibiotics were examined against these isolates, including amikacin, ciprofloxacin, levofloxacin, gentamicin (or tobramycin), colistin (or polymyxin B), and tigecycline. The susceptibility to tigecycline was found to be the highest $(413 / 439,94.1 \%)$, followed by colistin (or polymyxin B) $(579 / 690,83.9 \%)$, and amikacin $(298 / 885,33.7 \%$ ). Low susceptibilities were revealed for ciprofloxacin (10/630, 1.6\%), levofloxacin $(12 / 713$, $1.7 \%$ ), and gentamicin (or tobramycin) (11/630, 1.7\%). In Taiwan, Chiu et al. collected 457 isolates of CRKP from 21 hospital between Jan 2012 and Aug 2015 [28], and antimicrobial susceptibility testing of 19 agents against the 457 isolates was performed. Among agents they tested, only amikacin, colistin, and tigecycline showed high potencies, with susceptibilities of $78.8 \%$ (360/457), 85.6\% (391/457), and 88.6\% (405/457), respectively. According to a recent study from Iran, 50 isolates of CRKP were examined against 15 agents and were revealed with an $85 \%$ susceptibility rate against only amikacin and a high resistance rate against the other antimicrobial agents [31]. In this study, tigecycline demonstrated the highest susceptibility $(91.7 \%, 11 / 12)$ among 19 agents, followed by amikacin $(66.7 \%, 8 / 12)$ and gentamicin $(41.7 \%, 5 / 12)$, highlighting an urgent need for novel agents for CRKP.

Owing to the possibility of the horizontal transfer, the plasmid-mediated carbapenem resistance, namely the carbapenemase (gene), has been regarded as a critical mechanism needed to be monitored $[32,33]$. A previous study on carbapenemase-producing CRE isolates between 2013 and 2016 in the USA illustrated a diversity of carbapenemase genes, with $b l a_{\mathrm{OXA}-48}(25.0 \%, 6 / 24), b l a_{\mathrm{KPC}}(20.8 \%, 5 / 24), b l a_{\mathrm{NDM}}(20.8 \%, 5 / 24), b l a_{\mathrm{SME}}(20.8 \%$, $5 / 24), b l a_{\mathrm{IMP}}(8.3 \%, 2 / 24)$, and bla $_{\mathrm{VIM}}(4.2 \%, 1 / 24)$ [33]. A molecular epidemiological study from Europe revealed that a $b l a_{\mathrm{KPC}}$-like gene was the dominant carbapenemase gene in Italy, Greece, Portugal, Israel, and the UK, with a $b l a_{\mathrm{NDM}}-$ like gene in Serbia. However, the dominant carbapenemase gene in Turkey, Spain, Romania, and Belgium was a bla $a_{\mathrm{OXA}-48^{-}}$ like gene [34]. In a report from the Middle East, Alizadeh et al. described that bla OXA-48 was the most prevalent carbapenemase gene among 50 CRKP isolates $(78 \%, 39 / 50)$, followed by $b l a_{\mathrm{NDM}}(48 \%, 24 / 50), b l a_{\mathrm{IMP}}(22 \%, 11 / 50)$, bla $a_{\mathrm{VIM}}(12 \%, 6 / 50)$, and $b l a_{\mathrm{KPC}}(8 \%, 4 / 50)$ [31]. 
In the present work, among the $12 \mathrm{CRKP}$ isolates we examined, 7 isolates harbored the $b l a_{\mathrm{KPC}}$ gene and 1 isolate carried the $b l a_{\mathrm{OXA}-48}$ gene. Our observations were in agreement with the previous work conducted in Taiwan, with also bla $a_{\mathrm{KPC}}$ as the dominant gene [28].

Since carbapenem resistance worsened, novel therapeutic options were urgently requested to address the critical public health issue [35]. To this end, some $\beta$-lactam- $\beta$ lactamase inhibitor combination therapies were introduced into clinical settings recently, such as meropenem-vaborbactam, imipenem-relebactam, and ceftazidime-avibactam [7-10]. Moreover, combinations of azidothymidine (AZT), an antiviral agent usually used against HIV infection, plus other agents, were also reported, including AZT-colistin and AZTfosfomycin [11-13]. Hu et al. obtained 74 antibiotic-resistant Enterobacteriaceae strains from hospitals in 9 countries, including 23 ESBL-producing E. coli, 31 ESBL, 7 NDM-1-producing strains, and $13 \mathrm{mcr}-1$-positive E. coli [11]. The checkerboard analysis revealed $60.9 \%(14 / 23)$, 87.1\% (27/31), 100\% (7/7), and 92.31\% (12/13) synergetic effects in ESBL-producing E. coli, ESBL K. pneumoniae, NDM-1-producing strains, and mor-1-positive E. coli, respectively. In another evaluation conducted in Taiwan, Chang et al. reported 100\% synergistic activities for both KPC-producing and non-KPC-producing colistin-resistant CRKP isolates [12]. In vivo results demonstrated an extended lifespan and decreased risk ratios in a Caenorhabditis elegans model infected by a KPC-producing colistin-resistant CRKP isolate, positive support that AZTcolistin possessed the potential for treating CRKP infections. In a separate work, fosfomycin was noticed to have synergistic effects with AZT against 16 CRKP isolates, with the FICI of $87.5 \%$ isolates $(14 / 16) \leq 0.5$ [13]. In vivo, the larvae of Galleria mellonella were infected by KPC-producing or NDM-producing CRKP isolate and treated with AZT-fosfomycin combination therapy. Compared to AZT or fosfomycin single-agent therapy, greater survival curves were observed for AZT-fosfomycin combination therapy, with $20 \%$ to $50 \%$ higher survival rates. In our work, significantly decreased MIC distributions were observed for AS101-AZT combination (both $p<0.0001$ ), with $100 \%$ of synergistic activities against 12 CRKP isolates.

\section{Materials and Methods}

\subsection{Bacteria Isolates}

Twelve isolates of carbapenem-resistant K. pneumoniae (CRKP) were collected between 2013 and 2014 from 5 hospitals in a nationwide surveillance in Taiwan [28]. Carbapenem resistance was defined as being resistant to one of the carbapenems in accordance with the criteria recommended by the Clinical and Laboratory Standards Institute (CLSI) [36]. Among the 12 isolates, the main isolation source was urine (5/12, 41.7\%), followed by sputum $(4 / 12,33.3 \%)$, abscess $(1 / 12,8.3 \%)$, blood $(1 / 12,8.3 \%)$, deep wound $(1 / 12,8.3 \%)$ (Table 4 ). The CRKP isolates were stored at $-80^{\circ} \mathrm{C}$. Before the experiment, the isolates were recovered onto blood agar plate (Creative Media Plate, New Taipei, Taiwan) and cultured in the incubator at $37^{\circ} \mathrm{C}$. Colonies were subcultured onto fresh blood agar plate for stabilizing the physiological characteristics.

Table 4. Isolation sources of 12 isolates.

\begin{tabular}{cc}
\hline Isolation Source & No. of Isolates (\%) \\
\hline Abscess & $1(8.3)$ \\
Blood & $1(8.3)$ \\
Sputum & $4(33.3)$ \\
Urine & $5(41.7)$ \\
Deep wound & $1(8.3)$ \\
\hline
\end{tabular}

\subsection{Antimicrobial Susceptibility}

Susceptibilities of 19 antimicrobial agents against the 12 isolates were determined with a standard broth microdilution method following CLSI guidelines [36]. Briefly, two-fold serial dilutions of 19 antimicrobial agents were prepared. The bacterial suspension containing $2 \times 10^{8} \mathrm{CFU} / \mathrm{mL}$ of cells was prepared in brain heart infusion (BHI) broth (Becton Dickinson (BD), Sunnyvale, CA, USA) and diluted for 200 folds. The well-prepared drug 
solution and the diluted bacterial suspension were added into 96-well plate, respectively. The final bacterial density was $5 \times 10^{5} \mathrm{CFU} / \mathrm{mL}$. After 16-18 h incubation, the result was detected by SpectraMax Absorbance Reader (CMax Plus, Molecular Devices, LCC, Sunnyvale, CA, US) at $600 \mathrm{~nm}$. Among the 19 agents examined in this study, five classes of antibiotics were involved in the examination, including $\beta$-lactams and monobactam (ampicillin, ceftazidime, cefazolin, cefepime, cefoxitin, imipenem, ceftriaxone, meropenem, doripenem, ertapenem, cefotaxime, piperacillin-tazobactam, and aztreonam), aminoglycosides (amikacin and gentamicin), quinolones (ciprofloxacin and levofloxacin), inhibitors for folate synthesis (trimethoprim/sulfamethoxazole), and tetracycline (tigecycline). The results were also interpreted according to the breakpoints indicated by CLSI [36], and the interpretation for tigecycline was based on the guideline recommended by EUCAST [37]. Furthermore, the MICs for AS101 and azidothymidine (AZT) were also determined using the aforementioned broth microdilution method, and the results were collected for further evaluating the combination therapy of AS101 and AZT.

\subsection{Synergistic Analysis}

To investigate the synergistic effects between AZT (Toronto Research Chemicals, Toronto, Canada) and AS101 (Development Center for Biotechnology, Taipei, Taiwan), a checkerboard method was applied as described in a previous study [12,38]. In short, bacterial suspension containing $2 \times 10^{8} \mathrm{CFU} / \mathrm{mL}$ of cells was prepared in brain heart infusion (BHI) broth (Becton Dickinson (BD), Sunnyvale, CA, USA) and subsequently diluted to an appropriate concentration. Two-fold serial dilution of AZT and AS101 were also prepared in the appropriate ranges of concentrations, and the well-prepared AZT and AS101 solutions (50 $\mu \mathrm{L}$ each) were added into a 96-well plate. One hundred $\mu \mathrm{L}$ of the diluted bacterial suspension was also dispensed into wells at a final bacterial concentration of $5 \times 10^{5} \mathrm{CFU} / \mathrm{mL}$. The result was also detected by SpectraMax Absorbance Reader (CMax Plus, Molecular Devices, LCC, Sunnyvale, CA, US) at $600 \mathrm{~nm}$. The fractional inhibitory concentration (FIC) index was calculated to determine the synergistic effects as the following formula

$$
\mathrm{FIC}=\frac{\mathrm{MIC}_{\mathrm{AS} 101} \text { in combination }}{\mathrm{MIC}_{\mathrm{AS} 101} \text { in sin gle }}+\frac{\mathrm{MIC}_{\mathrm{ZDV}} \text { in combination }}{\mathrm{MIC}_{\mathrm{ZDV}} \text { in single }}
$$

Generally, the interaction between two agents was defined as synergism if FIC index $\leq 0.5$; it was interpreted as interaction while the FIC index was between 0.5 and 4; antagonism was defined as an FIC index $>4$.

\subsection{Polymerase Chain Reaction Detection}

Extended-spectrum $\beta$-lactamase (ESBL) genes $\left(b l a_{\mathrm{SHV}}, b l a_{\mathrm{TEM}}, b l a_{\mathrm{OXA}}, b l a_{\mathrm{CTX}-\mathrm{M}-\mathrm{G} 1}\right.$, $b l a_{\mathrm{CTX}-\mathrm{M}-\mathrm{G} 2}$, and $\left.b l a_{\mathrm{CTX}-\mathrm{M}-\mathrm{G} 9}\right)$, plasmid-mediated AmpC genes (bla $a_{\mathrm{DHA}}$ and $\left.b l a_{\mathrm{CMY}}\right)$, carbapenemase genes $\left(b l a_{\mathrm{KPC}}, b l a_{\mathrm{NDM}}, b l a_{\mathrm{IMP}}, b l a_{\mathrm{NMC}}, b l a_{\mathrm{SME}}, b l a_{\mathrm{VIM}}, b l a_{\mathrm{SPM}-1}, b l a_{\mathrm{GIM}-1}, b l a_{\mathrm{SIM}-1}\right.$, $b l a_{\mathrm{IMI}}, b l a_{\mathrm{GES}}$, and $\left.b l a_{\mathrm{OXA}-48}\right)$, plasmid-mediated colistin-resistant gene ( $\left.m c r-1\right)$ and outer membrane porin genes (ompK35 and ompK36) were detected as in our previous study [12]. All the polymerase chain reactions were performed using TaKaRa Taq ${ }^{\mathrm{TM}}$ (Cat. R001A, Takara Shuzo Co., Ltd., Tokyo, Japan) according to the instruction manual. Lastly, electrophoresis was applied to check PCR reactions, and DNA sequencing was utilized to validate the results, serviced by Genomics BioSci \& Tech Co., Ltd. (New Taipei, Taiwan).

\subsection{Statistical Analyses}

The MIC distribution graphs of AS101 and AZT, in combination or alone, were constructed by GraphPad Prism (v9.0, CA, USA) and analyzed with paired Student's t-test.

\section{Conclusions}

In this study, the noticeable changes in the MIC values after combining two drugs and fractional inhibitory concentration (FIC) index $\leq 0.5$ in all strains indicated the synergistic 
activities between AS101 and azidothymidine against 12 clinical carbapenem-resistant $K$. pneumoniae (CRKP) isolates, harboring the carbapenemase genes or ESBL genes. Taken together, our efforts provided a new insight to develop a novel therapeutic option. Even though the sample size was small, our work still provided a proof of concept for the combination of AZT and AS101. Further in vivo studies and large-scale evaluations are needed for AS101-AZT combination therapy in future study.

Supplementary Materials: The following are available online at https: / www.mdpi.com/article / 10.3390/pathogens10121552/s1, Figure S1: Growth curve of CRE-1044 in $16 \mu \mathrm{g} / \mathrm{mL}$ AS101 and $0.0625 \mu \mathrm{g} / \mathrm{mL}$ AZT.

Author Contributions: Conceptualization, S.-P.T.; methodology, W.-C.H., T.-Y.Y. and L.-C.W.; formal analysis, C.-L.S. and L.L.; resources, P.-L.L. and S.-P.T.; writing-original draft preparation, C.-L.S.; writing-review and editing, T.-Y.Y. and S.-P.T.; visualization, C.-L.S.; supervision, S.-P.T.; project administration, S.-P.T.; funding acquisition, S.-P.T. All authors have read and agreed to the published version of the manuscript.

Funding: This work was supported by the Ministry of Science and Technology of Taiwan (MOST 109-2320-B-037-027), Kaohsiung Medical University Drug Development and Value Creation Research Center (KMU-TC109A03-6), and National Sun Yat-sen University-Kaohsiung Medical University (NSYSU-KMU) Joint Research Project (NSYSUKMU 109-I005 and 110-I004).

Institutional Review Board Statement: Not applicable.

Informed Consent Statement: Not applicable.

Conflicts of Interest: The authors declare no conflict of interest.

\section{References}

1. Global Priority List of Antibiotic-Resistant Bacteria to Guide Research, Discovery and Development of New Antibiotics. Available online: https:/ / www.who.int/medicines/publications/WHO-PPL-Short_Summary_25Feb-ET_NM_WHO.pdf (accessed on 10 September 2021).

2. Tiri, B.; Sensi, E.; Marsiliani, V.; Cantarini, M.; Priante, G.; Vernelli, C.; Martella, L.A.; Costantini, M.; Mariottini, A.; Andreani, P.; et al. Antimicrobial Stewardship Program, COVID-19, and Infection Control: Spread of Carbapenem-Resistant Klebsiella Pneumoniae Colonization in ICU COVID-19 Patients. What Did Not Work? J. Clin. Med. 2020, 9, 2744. [CrossRef]

3. Nordmann, P.; Poirel, L. Epidemiology and Diagnostics of Carbapenem Resistance in Gram-negative Bacteria. Clin. Infect. Dis. 2019, 69, S521-S528. [CrossRef] [PubMed]

4. World Health Organization; European Centre for Disease Prevention and Control. Antimicrobial Resistance in the EU/EEA (EARS-Net)_Annual Epidemiological Report 2019. Available online: https://www.ecdc.europa.eu/en/publications-data/ surveillance-antimicrobial-resistance-europe-2019\#copy-to-clipboard (accessed on 10 September 2021).

5. Mendes, R.; Mendoza, M.; Singh, K.K.B.; Castanheira, M.; Bell, J.M.; Turnidge, J.D.; Lin, S.S.F.; Jones, R.N. Regional Resistance Surveillance Program Results for 12 Asia-Pacific Nations (2011). Antimicrob. Agents Chemother. 2013, 57, 5721-5726. [CrossRef] [PubMed]

6. Lee, Y.-L.; Lu, M.-C.; Shao, P.-L.; Lu, P.-L.; Chen, Y.-H.; Cheng, S.-H.; Ko, W.-C.; Lin, C.-Y.; Wu, T.-S.; Yen, M.-Y.; et al. Nationwide surveillance of antimicrobial resistance among clinically important Gram-negative bacteria, with an emphasis on carbapenems and colistin: Results from the Surveillance of Multicenter Antimicrobial Resistance in Taiwan (SMART) in 2018. Int. J. Antimicrob. Agents 2019, 54, 318-328. [CrossRef]

7. Novelli, A.; Del Giacomo, P.; Rossolini, G.M.; Tumbarello, M. Meropenem/vaborbactam: A next generation $\beta$-lactam $\beta$-lactamase inhibitor combination. Expert Rev. Anti-Infect. Ther. 2020, 18, 643-655. [CrossRef]

8. Lai, C.-C.; Chen, C.-C.; Tang, H.-J. Meropenem-Vaborbactam in the Treatment of Acute Bacterial Infections. J. Clin. Med. 2019, 8, 1650. [CrossRef]

9. Pogue, J.; Bonomo, R.A.; Kaye, K.S. Ceftazidime/Avibactam, Meropenem/Vaborbactam, or Both? Clinical and Formulary Considerations. Clin. Infect. Dis. 2018, 68, 519-524. [CrossRef] [PubMed]

10. Zhanel, G.G.; Lawrence, C.; Adam, H.; Schweizer, F.; Zelenitsky, S.; Zhanel, M.; Lagacé-Wiens, P.; Walkty, A.; Denisuik, A.; Golden, A.; et al. Imipenem-Relebactam and Meropenem-Vaborbactam: Two Novel Carbapenem- $\beta$-Lactamase Inhibitor Combinations. Drugs 2017, 78, 65-98. [CrossRef]

11. Hu, Y.; Liu, Y.; Coates, A. Azidothymidine Produces Synergistic Activity in Combination with Colistin against Antibiotic-Resistant Enterobacteriaceae. Antimicrob. Agents Chemother. 2019, 63, e01630-18. [CrossRef]

12. Chang, Y.-T.; Yang, T.-Y.; Lu, P.-L.; Lin, S.-Y.; Wang, L.-C.; Wang, S.-F.; Hsieh, Y.-J.; Tseng, S.-P. Combination of Colistin and Azidothymidine Demonstrates Synergistic Activity against Colistin-Resistant, Carbapenem-Resistant Klebsiella pneumoniae. Microorganisms 2020, 8, 1964. [CrossRef] 
13. Antonello, R.M.; Di Bella, S.; Betts, J.; La Ragione, R.; Bressan, R.; Principe, L.; Morabito, S.; Gigliucci, F.; Tozzoli, R.; Busetti, M.; et al. Zidovudine in synergistic combination with fosfomycin: An in vitro and in vivo evaluation against multidrug-resistant Enterobacterales. Int. J. Antimicrob. Agents 2021, 58, 106362. [CrossRef] [PubMed]

14. Shaffer, N.; Chuachoowong, R.; Mock, P.A.; Bhadrakom, C.; Siriwasin, W.; Young, N.L.; Chotpitayasunondh, T.; Chearskul, S.; Roongpisuthipong, A.; Chinayon, P.; et al. Short-course zidovudine for perinatal HIV-1 transmission in Bangkok, Thailand: A randomised controlled trial. Lancet 1999, 353, 773-780. [CrossRef]

15. Loose, M.; Naber, K.G.; Hu, Y.; Coates, A.; Wagenlehner, F.M. Urinary bactericidal activity of colistin and azidothymidine combinations against mcr-1-positive colistin-resistant Escherichia coli. Int. J. Antimicrob. Agents 2019, 54, 55-61. [CrossRef] [PubMed]

16. Shahbazi, F.; Dashti-Khavidaki, S. Colistin: Efficacy and safety in different populations. Expert Rev. Clin. Pharmacol. 2015, 8, 423-448. [CrossRef] [PubMed]

17. Sredni, B.; Caspi, R.; Klein, A.; Kalechman, Y.; Danziger, Y.; BenYa'Akov, M.; Tamari, T.; Shalit, F.; Albeck, M. A new immunomodulating compound (AS-101) with potential therapeutic application. Nature 1987, 330, 173-176. [CrossRef] [PubMed]

18. Vázquez-Tato, M.P.; Mena-Menéndez, A.; Feás, X.; Seijas, J.A. Novel Microwave-Assisted Synthesis of the Immunomodulator Organotellurium Compound Ammonium Trichloro(dioxoethylene-O,O')tellurate (AS101). Int. J. Mol. Sci. 2014, 15, 3287-3298. [CrossRef] [PubMed]

19. Halpert, G.; Sredni, B. The effect of the novel tellurium compound AS101 on autoimmune diseases. Autoimmun. Rev. 2014, 13, 1230-1235. [CrossRef]

20. Vonsover, A.; Loya, S.; Sredni, B.; Albeck, M.; Gotlieb-Stematsky, T.; Araf, O.; Hizi, A. Inhibition of the Reverse Transcriptase Activity and Replication of Human Immunodeficiency Virus Type 1 by AS 101 In Vitro. AIDS Res. Hum. Retrovir. 1992, 8, 613-623. [CrossRef]

21. Indenbaum, V.; Bin, H.; Makarovsky, D.; Weil, M.; Shulman, L.; Albeck, M.; Sredni, B.; Mendelson, E. In vitro and in vivo activity of AS101 against West Nile virus (WNV). Virus Res. 2012, 166, 68-76. [CrossRef]

22. Yang, T.-Y.; Tseng, S.-P.; Dlamini, H.N.; Lu, P.-L.; Lin, L.; Wang, L.-C.; Hung, W.-C. In Vitro and In Vivo Activity of AS101 against Carbapenem-Resistant Acinetobacter baumannii. Pharmaceuticals 2021, 14, 823. [CrossRef]

23. Yang, T.-Y.; Kao, H.-Y.; Lu, P.-L.; Chen, P.-Y.; Wang, S.-C.; Wang, L.-C.; Hsieh, Y.-J.; Tseng, S.-P. Evaluation of the Organotellurium Compound AS101 for Treating Colistin- and Carbapenem-Resistant Klebsiella pneumoniae. Pharmaceuticals 2021, 14, 795. [CrossRef]

24. Daniel-Hoffmann, M.; Albeck, M.; Sredni, B.; Nitzan, Y. A potential antimicrobial treatment against ESBL-producing Klebsiella pneumoniae using the tellurium compound AS101. Arch. Microbiol. 2009, 191, 631-638. [CrossRef] [PubMed]

25. Daniel-Hoffmann, M.; Sredni, B.; Nitzan, Y. Bactericidal activity of the organo-tellurium compound AS101 against Enterobacter cloacae. J. Antimicrob. Chemother. 2012, 67, 2165-2172. [CrossRef]

26. Patel, G.; Huprikar, S.; Factor, S.H.; Jenkins, S.G.; Calfee, D.P. Outcomes of Carbapenem-Resistant Klebsiella pneumoniae Infection and the Impact of Antimicrobial and Adjunctive Therapies. Infect. Control. Hosp. Epidemiol. 2008, 29, 1099-1106. [CrossRef]

27. Antibiotic Resistance Threats in the United States. 2013. Available online: https://www.cdc.gov/drugresistance/pdf/ar-threats2013-508.pdf (accessed on 10 September 2021).

28. Chiu, S.-K.; Ma, L.; Chan, M.-C.; Lin, Y.-T.; Fung, C.-P.; Wu, T.-L.; Chuang, Y.-C.; Lu, P.-L.; Wang, J.-T.; Lin, J.-C.; et al. Carbapenem Nonsusceptible Klebsiella pneumoniae in Taiwan: Dissemination and Increasing Resistance of Carbapenemase Producers During 2012-2015. Sci. Rep. 2018, 8, 8468. [CrossRef] [PubMed]

29. Van Duin, D.; Perez, F.; Rudin, S.D.; Cober, E.; Hanrahan, J.; Ziegler, J.; Webber, R.; Fox, J.; Mason, P.; Richter, S.S.; et al. Surveillance of Carbapenem-Resistant Klebsiella pneumoniae: Tracking Molecular Epidemiology and Outcomes through a Regional Network. Antimicrob. Agents Chemother. 2014, 58, 4035-4041. [CrossRef]

30. Han, J.H.; Goldstein, E.J.; Wise, J.; Bilker, W.B.; Tolomeo, P.; Lautenbach, E. Epidemiology of Carbapenem-Resistant Klebsiella pneumoniae in a Network of Long-Term Acute Care Hospitals. Clin. Infect. Dis. 2016, 64, 839-844. [CrossRef] [PubMed]

31. Alizadeh, N.; Rezaee, M.A.; Kafil, H.S.; Hasani, A.; Barhaghi, M.H.S.; Milani, M.; Sefidan, F.Y.; Memar, M.Y.; Lalehzadeh, A.; Ghotaslou, R. Evaluation of Resistance Mechanisms in Carbapenem-Resistant Enterobacteriaceae. Infect. Drug Resist. 2020, 13, 1377-1385. [CrossRef]

32. Elshamy, A.A.; Aboshanab, K.M. A review on bacterial resistance to carbapenems: Epidemiology, detection and treatment options. Futur. Sci. OA 2020, 6, FSO438. [CrossRef]

33. Codjoe, F.S.; Donkor, E.S. Carbapenem Resistance: A Review. Med. Sci. 2017, 6, 1. [CrossRef]

34. David, S.; Reuter, S.; Harris, S.R.; Glasner, C.; Feltwell, T.; Argimon, S.; Abudahab, K.; Goater, R.; Giani, T.; Errico, G.; et al. Epidemic of carbapenem-resistant Klebsiella pneumoniae in Europe is driven by nosocomial spread. Nat. Microbiol. 2019, 4, 1919-1929. [CrossRef] [PubMed]

35. Doi, Y. Treatment Options for Carbapenem-resistant Gram-negative Bacterial Infections. Clin. Infect. Dis. 2019, 69, S565-S575. [CrossRef] [PubMed]

36. Clinical and Laboratory Standards Institute. Performance Standards for Antimicrobial Susceptibility Testing: Thirtieth Informational Supplement; Document M100-S130 CLSI; Clinical and Laboratory Standards Institute: Wayne, PA, USA, 2020. 
37. The European Committee on Antimicrobial Susceptibility Testing. Breakpoint tables for interpretation of MICs and Zone Diameters. Version 11.0, 2021. Available online: http:/ / www.eucast.org (accessed on 10 September 2021).

38. Tseng, S.-P.; Wang, S.-F.; Ma, L.; Wang, T.-Y.; Yang, T.-Y.; Siu, L.K.; Chuang, Y.-C.; Lee, P.-S.; Wang, J.-T.; Wu, T.-L.; et al. The plasmid-mediated fosfomycin resistance determinants and synergy of fosfomycin and meropenem in carbapenem-resistant Klebsiella pneumoniae isolates in Taiwan. J. Microbiol. Immunol. Infect. 2017, 50, 653-661. [CrossRef] [PubMed] 